\title{
¿Pensamiento crítico? Ni lo uno ni lo otro*
}

\author{
Joan Manuel Madrid Hincapié \\ Fundación Universitaria Claretiana, Uniclaretiana, Medellín, Colombia. \\ joan.madrid@uniclaretiana.edu.co \\ http://orcid.org/0000-0003-0838-6510
}

\section{RESUIMEN}

En el presente escrito de reflexión se PISA del año 2018 y los modos de proceder plantea que una de las responsabilidades asignadas al educando es aportar al desarrollo del pensamiento crítico, la cual está contemplada en la Ley General de Educación 115 de 1994. Por ello, según una cuantiosa producción académica, se han podido mejorar los niveles de comprensión lectora y de competencia escritural en breves lapsos. Pero esto termina siendo contradictorio si se tienen en cuenta los recientes puntajes obtenidos en las pruebas de la población colombiana en el presente confinamiento obligatorio, lo cual demuestra una escasa correspondencia con dicha capacidad intelectual. De allí que tales resultados sean cantinflescos para una condición que requiere mucho más que la lectura y la escritura.

Palabras clave: pensamiento crítico; práctica pedagógica; didáctica de las ciencias sociales; lineamientos curriculares; estándares de competencias.

Cómo citar: Madrid, J. (2020). ¿Pensamiento crítico? Ni lo uno ni lo otro. Ciencias Sociales y Educación, 9(18), 159-174, https://doi.org/10.22395/csye.v9n18a7

Recibido: 1 de julio de 2020.

Aprobado: 24 de julio de 2020. 


\section{Critical Thinking? Neither Critical nor Thinking}

\section{ABSTRACT}

This reflection article states that one of the responsibilities assigned to the pupils is contributing to critical thinking development, which is contemplated in the General law for Education 115th of 1994. For that, according to a numerous academic production, there has been a possibility for improving the levels of reading comprehension and writing competence in brief periods. But this ends up being contradictory when contrasted with the recent scores obtained in the PISA texts of 2018 and the ways of facing the mandatory confinement by the Colombian population, which shows a scarce correspondence with the said intellectual capacity. That is why those results are a mockery for something that requires much more than reading and writing.

Keywords: critical thinking; teaching practice; didactics in social sciences; curricular guidelines; competencies standards.

\section{Pensamento crítico? Nem um nem outro}

\section{RESUMO}

Nesta reflexão, afirma-se que uma das responsabilidades atribuídas ao aluno é contribuir para o desenvolvimento do pensamento crítico, isso está previsto na Lei Geral de Educação 115 de 1994. Portanto, de acordo com uma vasta produção acadêmica, pôde-se melhorar os níveis de compreensão de leitura e competência de escrita em curtos períodos de tempo. Mas isso acaba sendo contraditório se considerarmos as notas obtidas no Programa Internacional de Avaliação de Estudantes (Pisa, na sigla em inglês) de 2018 e como a população colombiana vem agindo no atual confinamento obrigatório, o que mostra pouca correspondência com dita capacidade intelectual. Consequentemente, esses resultados são incoerentes quando a situação exige muito mais do que ler e escrever.

Palavras-chave: diretrizes curriculares; ensino de ciências sociais; padrões de competência; pensamento crítico; prática pedagógica. 


\section{Introducción}

La promulgación de la Ley 115 (1994) y posteriormente la Resolución 2343 (1996) determinaron los lineamientos curriculares y los estándares de competencias del área de Ciencias Sociales en la educación básica y media. Posteriormente, se promulgaron los derechos básicos de aprendizaje [DBA] (Ministerio de Educación Nacional [MEN], 2016), donde no solo se reconoce esta área como obligatoria y fundamental, sino que ratifican la importancia que tienen las distintas disciplinas del conocimiento que la integran y sus respectivos aportes teóricos. Con ello, se buscó que el estudiante pudiera asistir a una experiencia formativa donde aprehendiera a aproximarse a las problemáticas de su entorno mediante los procedimientos técnicos con que los profesionales de antropología, ciencia política, derecho, economía, demografía, estadística, sociología, entre otros, generan su conocimiento (MEN, 2004, 2002). Este hecho representa la intención de consolidar una educación científica que pueda responder a las necesidades de una sociedad como la colombiana.

Esta preparación, por supuesto, no puede incurrir en el equívoco de convertir las instituciones educativas en centros de formación de científicos sociales, ya que dicho propósito estaba determinado exclusivamente para la universidad (MEN, 2004) que, aparte de fortalecer la formación para la ciudadanía, la democracia y la memoria histórica, se enfoca en afianzar el desarrollo del pensamiento crítico. Para ello, es preciso que el estudiante se ejercite en el trabajo de recolección, manejo, análisis de datos, formulación de preguntas y nuevas hipótesis, contrastación y verificación de resultados en el marco de la deliberación académica para que, según el MEN (2002), este pueda comprender la realidad nacional y transformarla a partir de la misma. Asimismo, esta formación permitiría que, como ciudadano con conciencia crítica, participe activamente en la construcción de una convivencia respetuosa de la diferencia, solidaria, ecuánime y justa.

Sin embargo, llama la atención que la producción académica nacional e internacional sostenga que obtuvo importantes resultados en el desarrollo del pensamiento crítico en breves lapsos de tiempo, por lo regular delimitados a un año lectivo en los que se implementaron una serie de actividades de toda índole bajo el epíteto de la innovación, en lo que se encuentra el mejoramiento de la comprensión lectora y la habilidad escritural del estudiante y que según Nova (2020), Castro (2019), Sare (2019), Guerrero, Polo, Martínez y Ariza (2018), Reynosa (2018), Morales (2017), Rímac, Velásquez y Hernández (2017), García y Arrieta (2016), Santiago (2016), Mejía, López y Valenzuela (2015), García y Gómez (2015), tales progresos pudieron verse reflejadas en los puntajes alcanzados en pruebas estandarizadas como las Saber 11.. 
Estos resultados ejemplares contrastan con los puntajes alcanzados en las pruebas del Programa Internacional para la Evaluación de Estudiantes [PISA] aplicadas por la Organización para la Cooperación y el Desarrollo Económicos [OCDE] (2018). El país ocupó los últimos lugares debido a las serias falencias en la comprensión lectora en comparación con los puntajes obtenidos en el 2015. Además, la encuesta nacional de lectura [Enlec] (Departamento Administrativo Nacional de Estadística [DANE], 2017) reveló que los colombianos, después de finalizado el bachillerato, leen en promedio 5,1 libros al año. Este hecho corrobora lo planteado por la Red de Lectura y Escritura en Educación Superior [Redless] (2015) sobre las serias falencias que evidencian los estudiantes provenientes de los colegios. Según Redless (2015), aparte de las limitaciones ortográficas, no saben redactar textos críticos ni mucho menos cuentan con capacidades que les permita una óptima comprensión de documentos académicos. De hecho, esta es una de las principales causas de deserción en la educación superior.

García et al. (2015) aseveran que un sistema educativo que resulta poco atractivo para los mejores profesionales en términos de reconocimiento social y remuneración económica permite convalidar que el país está demasiado lejos de materializar el ideal de calidad educativa si su cuerpo docente acumula títulos de alto nivel sin que su estilo de enseñanza haya sido ajustado a las exigencias de la sociedad del conocimiento (Torrejano, 2015; García et al., 2013). En este contexto, resulta poco creíble que en un año lectivo un estudiante mejore su capacidad de pensar críticamente porque este demostró algún progreso en la comprensión lectora o en la realización de ejercicios escritos. Y es menos creíble si, pese a que hay una normatividad que procura consolidar un modelo de educación que responda a los requerimientos del actual contexto tecnológico, científico, político, económico y social, todavía persisten prácticas pedagógicas empeñadas en fortalecer el desempeño escolar a través de la transcripción de contenidos en el cuaderno de notas, la elaboración de mapas, maquetas, consultas bibliográficas, entre otros, sin que sus resultados sean sometidos a una revisión rigurosa de lo que realmente se aprende.

Aunado a ello, termina siendo cantinflesco que una capacidad intelectual como el pensamiento crítico se reduzca a la lectura y la escritura, y a su vez sea "medida" con pruebas estandarizadas. Esto devela, en primera instancia, el desconocimiento - ¿por acción u omisión? - de lo que implica lograr que una persona desarrolle dicha manera de pensar. A pesar de la cuantiosa literatura publicada al respecto, que ha atiborrado de definiciones tal capacidad sin posibilitar el consenso de lo que debe entenderse por esta, dejan de lado que lograr que un estudiante se caracterice por ser un pensador crítico al finalizar su educación básica demanda una preparación interdisciplinar. Pero sin la identificación del estilo y ritmo de aprendizaje de cada uno (Schunk, 2012), la 
caracterización del contexto sociofamiliar que facilita o complejiza el aprendizaje (Gardner, 2014), los fundamentos de la lógica y la lingüística para analizar argumentos (Bordes, 2011; Van Dijk, 2006; Saussure, 1994) y sin el conocimiento de qué tipo de pensamiento es el que se pretende desarrollar — si corresponde a la corriente cognitivista de razonamiento formal, a la Escuela de Frankfurt que procura la transformación de las problemáticas sociales o a la kantiana que pretende la consolidación del sapere aude (Hernández, 2019)—; no se logra desarrollar un pensamiento crítico.

A lo anterior se le suma un aspecto ignorado por tales trabajos que tiene que ver con lo subjetivo del asunto. Dicho de otro modo, mientras la teoría pedagógica asevera que la educación es un fenómeno social en el que intervienen una serie de circunstancias familiares, culturales, políticas, económicas y sociales — que en mayor o menor medida determinan el modo de asimilar los procesos con los que se busca lograr el aprendizaje (Huerta, 2015; Concepción y Rodríguez, 2014; Ortiz, 2013)—, termina siendo paradójico que la lectura o escritura de un documento se someta a una única perspectiva. Las anteriores particularidades, en concordancia con Gardner (2014), son las que posibilitan la diversidad de comprensiones ante una misma información. A juicio de Meseguer (2016), someter el pensamiento crítico al desarrollo de habilidades y destrezas lecto-escriturales devela el escaso conocimiento en cuanto al alcance de esta capacidad intelectual de orden superior. Un pensador crítico se distingue por un raciocinio independiente que evita repetir tópicos o postulados de moda, lo que le posibilita formular supuestos propios. De acuerdo con Van Gelder (2000), lograr pensar críticamente es una tarea compleja, difícil y ardua que demanda disciplina, constancia y estructurados hábitos de estudio.

\section{La escisión entre el país teórico y el país titulado}

En 1945, Jorge Eliécer Gaitán Ayala (1903-1948) declaró que en Colombia se evidencian dos países: el país político que concierne a una clase dirigente que procura fortalecer los mecanismos que le garanticen su permanencia en el poder, y el país nacional que corresponde a la clase obrera y campesina. Esta última se encuentra olvidada mientras busca cómo resolver sus necesidades económicas, superar sus limitaciones educativas, resolver sus dificultades de vivienda y sobrevivir al riesgo de la delincuencia. El país nacional permanece excluido, desatendido por ese país político que no podría existir sin el otro (Arias, 2015).

Para efectos del presente caso se hace un símil en el ámbito educativo donde se vislumbran, igualmente, dos países: el país teórico y el país titulado. El primero se refiere a la producción académica que señala los avances que se han venido dando en el desarrollo del pensamiento crítico a partir de la enseñanza 
de las Ciencias Sociales. El segundo se refiere a la cantidad de egresados que año tras año obtienen su título de bachiller, pero sus formas de afrontar las problemáticas sociales, resolver sus diferencias y proceder como ciudadanos en los escenarios que habitan, dan cuenta de una importante escisión que genera considerables dudas en cuanto a la confiabilidad de lo que menciona el primero en contraste con lo que practica el segundo.

Ahora bien, Rivas (2018) y Puyana (2014) señalan que Colombia no solo se ha convertido en el objeto de análisis por sus particularidades históricas, políticas, económicas, educativas, religiosas, étnicas, ideológicas y culturales, sino que además es un territorio reconocido por su diversidad. Esta característica le posibilita coconstruir un modelo de sociedad que sirva de referente para las naciones latinoamericanas. Por consiguiente, justifica que tal ventaja coadyuvará a que las futuras generaciones cuenten con las garantías que en la actualidad propician unas condiciones de vida óptimas en comparación con otros países latinoamericanos. Pero resulta incongruente que dichas condiciones sean escasamente tenidas en cuenta para consolidar un currículo crítico desde los planteamientos del país teórico para que el titulado pueda recibir una enseñanza de las Ciencias Sociales ajustada a la realidad colombiana, de manera que la escisión entre los dos países comience a reducirse.

En términos estratégicos, esta intencionalidad termina siendo ventajosa para un sistema educativo que, según la Constitución Política (1991), procura aportar al progreso económico, político y social del país a través del "acceso al conocimiento, a la ciencia, a la técnica y a los demás bienes y valores de la cultura" (art. 67). Además, en consonancia con la Ley 115 (1994), uno de los propósitos de la educación colombiana es el

desarrollo de la capacidad crítica, reflexiva y analítica que fortalezca el avance científico y tecnológico nacional, orientado con prioridad al mejoramiento cultural y de la calidad de vida de la población, a la participación en la búsqueda de alternativas de solución a los problemas y al progreso social y económico del país. (art. 5)

En este contexto, para un área como Ciencias Sociales, según el MEN (2002), es fundamental el proceso formativo. Este proceso constituye en el estudiante una manera de ver y comprender el mundo, de aproximarse a sus dinámicas, fenómenos y acontecimientos que estructuran la forma de relacionarse en el aspecto político, familiar, cultural, económico, ambiental, entre otros. Por ello, es importante consolidar un modelo de enseñanza que se convierta en un laboratorio para el estudio de las problemáticas sociales mediante los procedimientos técnicos con que los científicos construyen sus corpus teóricos, pero que se adecúen a las condiciones de tales niveles de enseñanza. El resultado final de todo el ciclo escolar sería un egresado capaz de pensar críticamente. 
Pero mientras el país teórico continúa aseverando notables avances en la formación de sujetos críticos gracias a la implementación de un variado número de estrategias cuyos resultados les convalida aseverar fehacientemente haber mejorado la calidad del aprendizaje del pensamiento crítico (Maturana y Lombo, 2020; Robles, 2019; Díaz, 2018; Ríos, 2017; Lara y Rodríguez, 2016; Mejía y Mejía, 2015; García, 2014; Reyes, Mellizo y Ortega, 2013; Bolaños, 2012), el país titulado se caracteriza por unas formas de proceder contrarias a lo que se supone fueron preparados en las instituciones educativas (La Patria, 2020; El Tiempo, 2020; Acero, 2019; Guerra, 2019; Isaza y Restrepo, 2018; Manchola, 2018; La W, 2017; Lozano, 2017; Noticias Telemedellín, 2017; Mundo Deportivo, 2016; Garzón, Cardona y Romero, 2015; Instituto Colombiano de Bienestar Familiar [ICBF], 2015; El Heraldo, 2014). Desde este punto de vista, parece que la realidad que menciona el primero corresponde a la intención de materializar esa sociedad ideal que debería ser Colombia. El segundo, entonces, evoca los postulados de Sorokin (1964), que dice que cuando una dificultad no puede ser resuelta, el mejor modo de solucionarla es con términos nuevos. Y considerando la sagacidad del colombiano, lo más viable es renombrar todas estas problemáticas como "retos" (Puyana, 2014).

\section{La sociedad colombiana: ireflejo de un sistema educativo que propende forjar el pensamiento crítico?}

Según Duque (2000), un país que históricamente ha estado atosigado de las más nefastas problemáticas, infamias e injusticias sociales, corrobora que sus modelos educativos continúan siendo desacertados, por no decir en permanente crisis, aunque sus diversas normatividades se hayan redactado para corregirlos de manera que pudiesen ser la respuesta a las dificultades que obstaculizan materializar la anhelada calidad de la educación colombiana. No obstante, ante los sucesos que componen la realidad educativa nacional, es pertinente retomar lo señalado por la Fundación Compartir (2014). A juzgar por los resultados obtenidos en las pruebas estandarizadas nacionales e internacionales, el sistema educativo colombiano poco ha logrado en la formación de un sujeto crítico capaz de aportar a la construcción de una sociedad más democrática. Basta con detenerse a observar la correspondencia entre lo que certifica el título de un grado y su forma de proceder en la vida cotidiana. Resulta incongruente que quien haya pasado por la escuela y culmine satisfactoriamente cada uno de sus ciclos según sus informes de desempeño académico, evidencie serias falencias en lo personal para interactuar y convivir en sociedad, según lo señalado en párrafos anteriores. 
Dicho de otra forma, es una sociedad cuya "capacidad de juicio" demuestra una inclinación por la cultura traqueta (Vega, 2015)릴 la naturalización del delito, el pillaje, la corrupción y el engaño, por mencionar algunas. Además, su historia ha sido construida por la violencia, el conflicto armado, las guerras intestinas, la discriminación política, económica, cultural, el escaso sentido de soberanía y de pertenencia por el territorio. Por ello, ante la pérdida del Canal de Panamá, la entrega de una porción de mar territorial en San Andrés y Providencia, los escándalos de Odebrecht, la comunidad del anillo, el cartel de la toga, el carrusel de la contratación; la población ha permanecido en silencio, apática e indiferente (Ospina, 2016), pero atenta a lo que ocurra en la vida de cantantes, jugadores de fútbol, modelos y reinas de belleza. Basta con recordar ciertos comportamientos de unos compatriotas en el último campeonato mundial de fútbol ${ }^{2}$ que dejan mucho que decir sobre el tipo de ciudadano que está egresando de las instituciones educativas del país.

La aprobación de cada año lectivo supone haber alcanzado los estándares de competencias establecidos para la educación básica y media. Esta aprobación se vincula a unos proyectos educativos institucionales [PEI] que, en la mayoría de los casos, se basan en modelos pedagógicos, cognitivistas, constructivistas, críticos, conceptuales y un considerable etcétera de denominaciones, sin dejar de lado la obligatoriedad de la cátedra de paz y la implementación de actividades que promueven la inclusión, la diversidad, el género, la prevención y mitigación de la violencia intrafamiliar y escolar. Sin embargo, la sociedad colombiana permanece impávida ante lo que acontece en el sector público y privado, por lo que la ejecución de los planes, programas, políticas y proyectos educativos públicos en poco o nada contribuyen al mejoramiento de las condiciones de vida. Antes bien, eximen al Estado de su responsabilidad. Según Tobón (2018) y Villamizar (2016), todo esto ocurre en Colombia porque su población ha sido preparada para naturalizar tales vejámenes con el fin de favorecer la permanencia del statu quo.

¿De qué manera se justifica que la población proceda de manera contraria a lo que establece la Constitución Política si recibir un título sustenta haberse formado bajo los preceptos que contempla una filosofía y un horizonte institucional? ¿Es posible creer que se desarrollaron las bases del pensamiento crítico en la educación básica y media, cuando la población egresada sucumbe ante las promesas de una vida mejor anunciadas por los medios de comunicación masiva? ¿Es posible creer que la escuela aporta al desarrollo de esta capacidad intelectual de orden superior, cuando sus titulados asumen una postura inerme

Según Vega (2005), lo "traqueto" corresponde al modelo de vida que posibilita las ganancias obtenidas del narcotráfico, cuyo principal ícono en Colombia fue Pablo Emilio Escobar Gaviria (1949-1993) y que se robustece gracias a la transmisión de series televisivas relacionadas con el tema.

$2 \quad \mathrm{Al}$ respecto véase Cablenoticias. (2018). 
ante los escenarios de participación política del país? Aunado a ello, ¿son convincentes unos resultados que aseveran importantes logros en el pensamiento crítico, pero estos sujetos reproducen ideas de moda o aceptan información sin el respectivo escrutinio para confirmar su validez? ¿En dónde pueden corroborarse, entonces, tales logros en el desarrollo del pensamiento crítico, dado el comportamiento asumido por los colombianos en el presente confinamiento obligatorio debido a la COVID-19?

\section{La noción de pensamiento crítico}

Hernández (2019), Ross y Gautreaux (2018), Suárez et al. (2018) sostienen que, debido a la polisemia del término pensamiento crítico, se dificulta un consenso que permita una mejor comprensión de sus condiciones y de su alcance. Esta escasa claridad convalida lo señalado por Naessens (2015), ya que la diversidad de comprensiones que se vienen suscitando en el ámbito académico en poco o nada contribuyen a solventar el asunto. Al contrario, incurre en que se denomine pensamiento crítico a todo lo que sucede en las aulas de clase solo porque el estudiante logró señalar algún planteamiento o contradicción distinta. Pero, en realidad, no todo lo que se menciona de forma oral o escrita puede considerarse como tal si se tiene en cuenta que esta capacidad involucra un trabajo mancomunado de tipo interdisciplinar.

La educación básica y media en Colombia se componen de once años lectivos que permitirían deducir que son "suficientes" para instaurar en el educando las bases conceptuales del pensamiento crítico. Asimismo, lo establecido en la normatividad educativa vigente procura materializar un modelo de educación que propenda por el progreso tecnológico, científico, político, económico y social del país. Además, hay unos lineamientos curriculares y unos estándares de competencias que explícita e implícitamente corroboran la finalidad de ajustar los PEI, transformar las prácticas pedagógicas y los métodos de evaluación a las condiciones de la realidad nacional. ¿Será, entonces, que los resultados obtenidos en pruebas estandarizadas dan cuenta de una problemática relacionada con la estructura conceptual del sistema educativo? ¿De allí se desprenderá la escasa correspondencia con los proyectos educativos institucionales que se empeñan en ofrecer una educación tradicional y asignaturista y, por consiguiente, consolidar un sistema institucional de evaluación del estudiante [SIEE] que solo tiene en cuenta el hacer?

¿O será, más bien, que con esto se está evidenciando la escasa disposición por parte de las personas que, de acuerdo con Arias (2014), imposibilita que cualquier documento oficial que pretende generar una transformación de la educación en Colombia sea vista como una amenaza que atenta contra la comodidad 
de hacer siempre lo mismo? Según Puyana (2014), este hábito ha sido recurrente en la idiosincrasia nacional, ya que es bastante común creerse acreedor de un beneficio con el más mínimo esfuerzo. Si educar se refiere a un proceso que debe propender por el desarrollo de todas las dimensiones del ser humano de manera que repercutan en la sociedad, entonces el escenario educativo colombiano, en términos de Moreno (2016) y Pérez (2010), ha fracasado en su intento de aportar al progreso de una sociedad como la colombiana. El confinamiento obligatorio también viene reluciendo los aspectos que se supone fueron trabajados en el ámbito escolar (Canal Institucional, 2020; Portafolio, 2020; El Espectador, 2020; Semana, 2020). En cuanto al pensamiento crítico, basta con revisar lo que se consigna en los foros de las redes sociales para deducir el nivel de desarrollo que se logró.

Se concibe que el pensamiento crítico es más que un conjunto de habilidades para resolver pruebas estandarizadas sobre el mejoramiento de los procesos lecto-escriturales y la destreza para analizar argumentos. El fundamento básico del pensamiento crítico es una condición particular derivada de condiciones históricas, culturales, familiares, económicas, religiosas, ideológicas y políticas. El pensamiento crítico sirve de fundamento para que en cada uno de los niveles de la educación básica primaria, secundaria y media, la enseñanza de las Ciencias Sociales le provean al educando el escenario formativo para que logre la coherencia entre lo que piensa, siente, dice y hace. De este modo, es un exabrupto seguir limitando esta capacidad intelectual a un examen que deja de lado la dimensión subjetiva del asunto, lo cual posibilita que toda información sea comprendida de diversas maneras.

Por esta razón, pensar de manera crítica en términos de Meseguer (2016), es un ejercicio paulatino que se logra poco a poco y dura toda la vida. De allí que sea valioso cuando recurre a la inteligencia y la emplea eficientemente. Este hecho convalida que su enseñanza y aprendizaje son complejos, arduos, rigurosos y exigentes, dado que su perfeccionamiento implica contar con el apoyo de otros que estén dispuestos a corregir y pulimentar su conocimiento y experticia pedagógica. Para ello, se ha dejado de lado la discusión en cuanto al tipo de docencia que se requiere en Colombia y la correspondencia de sus planes de formación profesional con la calidad de la enseñanza que imparten en la institución educativa.

\section{Consideración final}

Si bien el pensamiento crítico se ha convertido en una "competencia" fundamental en la educación actual, y aunque se cuente con una vasta literatura académica que la define de diversas maneras —en esencia, todas ellas se centran 
en la capacidad para valorar y someter a juicio ideas con el fin de fortalecerlas o descartarlas-, en la práctica se ha vuelto recurrente limitarla a la habilidad para comprender información y producir textos escritos, ya que solo se dirige a lo que viene evaluándose en las pruebas estandarizadas. En este sentido, se han dejado de lado otras condiciones que permean tales procedimientos, por ejemplo, la dimensión subjetiva del asunto. Al ser esta un resultado de las particularidades familiares, culturales, regionales, políticas, económicas y sociales, no puede esperarse que todo individuo comprenda la información de la misma manera y, por consiguiente, elija una única respuesta. En definitiva, esto va en contravía de que cada persona es única y, por tanto, su ritmo y estilo de aprendizaje también lo son.

De este modo, no cabe la menor duda de que los "avances" alcanzados en el desarrollo del pensamiento crítico solo tienen impacto dentro del ámbito escolar, pues con ellos el educando cumple con una actividad asignada. Además, hay que tener en cuenta que de eso depende la aprobación/reprobación del área, lo que desde esta perspectiva omite que dicha clase de capacidades demandan un proceso paulatino, riguroso, arduo y complementado en cada uno de los grados que estructuran la educación básica primaria, secundaria y media. Por esta razón, se cree que esta sea la causa de las actuaciones de una sociedad que, a pesar de contar con mejores niveles de educación y más posibilidades de acceso, lo "aprendido" en el aula de clase solo funciona para dicho lugar y no como una apuesta que ayude a resignificar el comportamiento en cada una de las interacciones por fuera de dicho entorno.

Por consiguiente, lo que el país teórico asevera en cuanto al progreso alcanzado en el desarrollo del pensamiento crítico, no es más que un resultado momentáneo, pasajero y, por tanto, no garantiza un óptimo desarrollo de tal capacidad intelectual, menos aún si se pretende lograrlo desde el área de Ciencias Sociales de manera aislada con respecto a otros campos del conocimiento, puesto que se requiere de un verdadero trabajo transversal. Si lo que realmente se pretende es que el educando aprenda a analizar información para resolver pruebas, entonces se hace preciso contar con los aportes de disciplinas como la lógica - que posibilita aproximarse a los fundamentos que rigen los procesos que estructuran el pensamiento y los métodos que coadyuvan a identificar la validez o rechazo de planteamientos (Bordes, 2011) - y la lingüística, que viabiliza su comprensión desde la relación de los discursos con la estructura social (Van Dijk, 2006).

Un último aspecto para considerar es que con la actual estructura del sistema educativo colombiano, la formación del educando se establece por periodos y un orden de contenidos que, en la medida de lo posible, deben abarcarse durante 
las semanas establecidas para cada uno de los mismos. Aunado a ello, hay un modelo de evaluación del aprendizaje que beneficia en gran manera la aprobación de las áreas/asignaturas con los requerimientos que exigen desarrollar el pensamiento crítico. Además, son poco creíbles los importantes avances en breves lapsos de tiempo, ya que el aprendizaje es una condición atemporal que implica reconocer que lo que está asimilando el estudiante no causa un mayor impacto en la sociedad. En este sentido, se considera pertinente centrar la discusión en torno a la formación de quien decide enseñar el área de Ciencias Sociales, pues es poco probable aportar al desarrollo de la capacidad intelectual en cuestión cuando la práctica pedagógica se centra en temáticas que no aproximan al estudiante al contexto social que habita, ni mucho menos resaltan cada una de sus particularidades históricas, culturales, familiares, económicas, religiosas, ideológicas y políticas, ya que el pensamiento crítico es precisamente una actitud singular.

\section{Referencias}

Acero, H. (2019). ¿Qué pasa con la seguridad ciudadana? https://pares.com.co/2019/10/14/que-pasacon-la-seguridad-ciudadana/.

Arias, D. H. (2014). ¿Qué cambia la educación? Políticas públicas y condiciones de los cambios educativos. Ediciones Unisalle.

Arias, R. (2015). 9 de abril de 1948 (7ª reimp.). Panamericana.

Asamblea Nacional Constituyente. (1991, 20 de julio). Constitución Política de Colombia. Gaceta Constitucional n. ${ }^{\circ}$ 116. https://www.funcionpublica.gov.co/eva/gestornormativo/norma. $\operatorname{php} ? \mathrm{i}=4125$

Bolaños, B. I. (2012). Pensamiento crítico: formar para atreverse [tesis de maestría, Universidad de San Buenaventura]. Repositorio Institucional.

Bordes, M. (2011). Las trampas de Circe: falacias lógicas y argumentación informal. Ediciones Cátedra.

Canal Institucional. (2020). Indisciplina social: alerta por rumbas por cuarentena. https://www.canalinstitucional.tv/noticias/rumbas-en-cuarentena-colombia.

Cablenoticias. (2018, 19 de junio). Indignación por vergonzoso comportamiento de hinchas colombianos en Rusia [archivo de video]. Youtube. https://www.youtube.com/watch?v=Z9d_PdzWUpo.

Castro, M. S. (2019). Uso de estrategias cognitivas y socio-afectivas para mejorar y desarrollar el pensamiento crítico de los docentes de educación secundaria en la I.E. Rafael Díaz de Moquegua [trabajo de grado pregrado, Universidad Nacional de San Agustín de Arequipa]. Repositorio Institucional. http://repositorio.unsa.edu.pe/handle/UNSA/8964

Concepción, M. R. y Rodríguez, F. (2014). Rol del profesor y sus estudiantes en el proceso de enseñanza aprendizaje. Editorial Uniautónoma.

Congreso de la República de Colombia. (1994, 8 de febrero). Ley 115. Por la cual se expide la ley general de educación. Diario Oficial 41214. https://www.funcionpublica.gov.co/eva/gestornormativo/ norma_pdf.php?i=292 
Departamento Administrativo Nacional de Estadística [DANE]. (2017). Encuesta nacional de lectura [Enlec]. https://www.dane.gov.co/files/investigaciones/boletines/enlec/bt-enlec-2017.pdf.

Díaz, M. A. (2018). Pensamiento crítico en el aprendizaje de las ciencias sociales a partir de prácticas de lectoescritura en política en grado once [tesis de maestría, Universidad Externado de Colombia]. Repositorio Institucional. https://bdigital.uexternado.edu.co/handle/001/943

Duque, J. (2000). La misión de educar. Ediciones EDUQUE.

El Espectador. (2020). ¿Por qué tanta gente sale a la calle a desafiar al coronavirus? https://www. elespectador.com/noticias/el-mundo/por-que-tanta-gente-sale-la-calle-desafiar-al-coronavirusarticulo-910586/.

El Heraldo. (2014). Pelea entre dos adolescentes queda filmada en video. Las amenazas se iniciaron por las redes sociales. https://www.elheraldo.co/bolivar/madre-de-familia-denuncia-caso-de-violenciaescolar-163394.

El Tiempo. (2020). Una mujer murió al enfrentarse con otra en Siloé. https://www.eltiempo.com/colombia/cali/murio-una-mujer-en-una-rina-en-siloe-472782.

Fundación Compartir. (2014). Tras la excelencia docente. Cómo mejorar la calidad de la educación para todos los colombianos. https://www.fundacioncompartir.org/pdf/Tras\%20la\%20excelencia\%20 docente\%20-\%20estudio\%20final.pdf.

García, M. G. (2014). Desarrollo del pensamiento crítico a través del currículo crítico. Revista de Educación, (6), 217-274. https://fh.mdp.edu.ar/revistas/index.php/r_educ/article/view/764/781

García, H. y Arrieta, A. (2016). Potencialización del pensamiento crítico a través de la hipotemetacomprensión textual (hmt). Cultura Educación y Sociedad, 7(2), 54-71. https://revistascientificas.cuc. edu.co/culturaeducacionysociedad/article/view/1102

García, M., Espinosa, J. R., Jiménez, F. y Parra, J. D. (2013). Separados y desiguales. Educación y clases sociales en Colombia. Centro de Estudios de Derecho, Justicia y Sociedad [Dejusticia].

García, M. y Gómez, J. (2015). Desde la didáctica no parametral... Estrategia pedagógica para desarrollar el pensamiento crítico. Revista Educación y Humanismo, 17(29), 186-201. http://dx.doi. org/10.17081/eduhum.17.29.1252

García, S., Maldonado, D., Perry, G., Rodríguez, C. y Saavedra, J. E. (2015). Resumen y precisiones sobre "Tras la excelencia docente: cómo mejorar la calidad de la educación para todos los colombianos". En J. Zubiría (Comp.), La calidad de la educación bajo la lupa (pp. 33-48). Magisterio Editorial.

Gardner, H. (2014). Estructuras de la mente. La teoría de las inteligencias múltiples (10ª reimp.). Fondo de Cultura Económica.

Garzón, A., Cardona, D. y Romero, Z. (2015). Convivencia y cultura ciudadana: perspectiva desde la ciudad heroica. Universidad Libre.

Guerra, M. R. (2019). El fenómeno de la delincuencia juvenil. https://congresovisible.uniandes.edu.co/ agora/post/el-fenomeno-de-la-delincuencia-juvenil/10387/.

Guerrero, H., Polo, S., Martínez, J. y Ariza, P. (2018). Trabajo colaborativo como estrategia didáctica para el desarrollo del pensamiento crítico. Opción, 34(86), 959-986. http://produccioncientificaluz. org/index.php/opcion/article/view/23867 
Hernández, J. C. (2019). 'Pensamiento crítico': un análisis filosófico del concepto. Scientia in Verba Magazine, 2(1), 18-30.

Huerta, M. (2015). La estrategia en el aprendizaje. Editorial Magisterio.

Instituto Colombiano de Bienestar Familiar [ICBF]. (2015). Adolescentes, jóvenes y delitos: "elementos para la comprensión de la delincuencia juvenil en Colombia". https://www.icbf.gov.co/sites/default/ files/delincuencia_juvenil_web.pdf.

Isaza, M. y Restrepo, V. (2018). Repudio por brutal agresión entre jóvenes en un colegio de Medellín. El Colombiano. http://www.elcolombiano.com/antioquia/seguridad/pelea-de-estudiantes-en-elinem-de-medellin-NB8113852.

La Patria. (2020). Mujer trans fue herida en riña, cerca al barrio El Nevado. https://www.lapatria.com/ sucesos/mujer-trans-fue-herida-en-rina-cerca-al-barrio-el-nevado-451221.

La W. (2017). Cúcuta: caso de violencia escolar se registró en colegio privado. http://www.wradio.com. co/noticias/regionales/cucuta-caso-de-violencia-escolar-se-registro-en-colegio-privado/20170421/ nota/3442454.aspx.

Lara, J. M. y Rodríguez, E. (2016). Fomento del desarrollo del pensamiento crítico en estudiantes de grado décimo desde situaciones cotidianas en la asignatura de Filosofía. Educación y Humanismo, 18(31), 343-357. https://doi.org/10.17081/eduhum.18.31.1383

Lozano, R. (2017). Matoneo escolar, el ejemplo empieza por casa. https://www.semana.com/opinion/ articulo/matoneo-escolar-en-colombia/529196.

Manchola, C. (2018). Delito juvenil: un problema 'mayor' en Colombia. https://www.diariodelhuila.com/ delito-juvenil-un-problema-mayor-en-colombia.

Maturana, G. A. y Lombo, M. L. (2020). Inteligencia naturalista: efectos sobre el pensamiento crítico y las necesidades de cognición. Praxis \& Saber, 11(25), 177-204. https://doi.org/10.19053/22160159. v11.n25.2020.9094

Mejía, N., López, M. G. y Valenzuela, J. R. (2015). Pensamiento crítico en profesores de educación secundaria: caracterización de la competencia en instituciones antioqueñas (Colombia). Revista Latinoamericana de Estudios Educativos, 45(3), 138-177. https://www.redalyc.org/articulo. oa?id $=27041543006$

Mejía, L. y Mejía, A. L. (2015). Relaciones entre pensamiento histórico y pensamiento crítico en la enseñanza de las ciencias sociales en estudiantes de educación básica secundaria. Revista Interamericana de Investigación, Educación y Pedagogía, 8(2), 413-436. https://doi.org/10.15332/ s1657-107X.2015.0002.10

Meseguer, J. (2016). Pensamiento crítico: una actitud. Unir Editorial.

Ministerio de Educación Nacional de Colombia. (1996, 5 de junio). Resolución 2343. Por la cual se adopta un diseño de lineamientos generales de los procesos curriculares del servicio público educativo y se establecen los indicadores de logros curriculares para la educación formal. http://bibliotecadigital.usb. edu.co/bitstream/10819/1079/1/Ministerio_de_Educacion_Resolucion_2343 junio_5_de_1996.pdf

Ministerio de Educación Nacional [MEN]. (2016). Derechos Básicos de Aprendizaje [DBA]. Ciencias sociales. http://aprende.colombiaaprende.edu.co/sites/default/files/naspublic/DBA_C.Sociales.pdf.

Ministerio de Educación Nacional [MEN]. (2004). Formar en ciencias: iel desafío! Lo que necesitamos saber y saber hacer. https://www.mineducacion.gov.co/1759/articles-81033_archivo_pdf.pdf. 
Ministerio de Educación Nacional [MEN]. (2002). Serie lineamientos curriculares Ciencias Sociales. http://www.mineducacion.gov.co/1621/articles-339975_recurso_1.pdf.

Morales, J. A. (2017). Pensamiento crítico y lectura en ciencias sociales. Revista Electrónica Calidad en la Educación Superior, 8(2), 265-282. https://doi.org/10.22458/caes.v8i2.1943

Moreno, R. (2016). La conjura de los ignorantes. De cómo los pedagogos han destruido la enseñanza (4a ed.). Pasos Perdidos.

Mundo Deportivo. (2016). Exfutbolista Jairo Castillo, detenido otra vez por conducir ebrio. https://www. mundodeportivo.com/futbol/20160918/41409393138/exfutbolista-jairo-castillo-detenido-otra-vezpor-conducir-ebrio.html.

Naessens, H. (2015). Comparación entre dos autores del pensamiento crítico: Jacques Boisvert y Richard Paul-Linda Elder. http://ri.uaemex.mx/bitstream/handle/20.500.11799/57993/CAP\%2010\%20 COMPARACION.pdf? sequence $=1$.

Noticias Telemedellín. (2017). El 30\% de los estudiantes en Colombia ha sido víctima de acoso escolar. https://telemedellin.tv/estudiantes-colombia-victima-acoso-escolar/209288/.

Nova, M. T. (2020). La enseñanza de la filosofía y desarrollo del pensamiento crítico en la secundaria [trabajo de grado pregrado, Universidad Santo Tomás de Aquino]. Craiusta. Centro de Recursos para el Aprendizaje y la Investigación. https://repository.usta.edu.co/handle/11634/22246?show =full

Organización para la Cooperación y el Desarrollo Económicos [OCDE]. (2018). Colombia. https://www. oecd.org/pisa/publications/PISA2018_CN_COL_ESP.pdf.

Ortiz, A. (2013). Modelos pedagógicos y teorías del aprendizaje. Ediciones de la U.

Ospina, W. (2016). ¿Dónde está la franja amarilla? (10ª reimp.). Penguin Random House.

Pérez, A. (2010). ¿Para qué educamos hoy? Filosofía de la educación para un nuevo mundo (2a ed.). Biblos.

Portafolio. (2020). Colombianos no se han adaptado a las medidas contra el coronavirus. https://www. portafolio.co/tendencias/colombianos-no-se-han-adaptado-a-las-medidas-contra-el-coronavirus-540383.

Puyana, G. (2014). ¿Cómo somos? Los colombianos. Reflexiones sobre nuestra idiosincrasia y cultura (3a reimp.). Panamericana.

Red de Lectura y Escritura en Educación Superior [Redless]. (2015). Formación inicial en lectura y escritura en la universidad: de la educación media al desempeño académico en la educación superior (20112015). https://ascun.org.co/uploads/default/networks/91d587f5c2ed79daa422b3f409dfbb33.pdf.

Reyes, J. A., Mellizo, N. A. y Ortega, A. (2013). Pensamiento crítico y rendimiento académico en contextos educativos rural y urbano. https://es.scribd.com/document/269405364/Pensamiento-Critico-yRendimiento-Academico-en-Contextos-Educativos-Rural-y-Urbano.

Reynosa, W. (2018). Pensamiento Crítico en los estudiantes de grado séptimo de la Institución Educativa el Madroño del municipio de Belalcázar - Caldas [tesis de maestría, Universidad de Manizales]. Ridum. Repositorio Institucional Universidad de Manizales. https://ridum.umanizales.edu.co/ xmlui/handle/20.500.12746/3804

Rímac, G. D., Velásquez, M. E. y Hernández, R. (2017). Estrategias innovadoras para contribuir al desarrollo del pensamiento crítico. Revista de Educación, 8(10), 31-60. https://fh.mdp.edu.ar/revistas/ index.php/r_educ/article/view/2040 
Ríos, O. (2017). Desarrollo de habilidades de pensamiento crítico en las ciencias sociales a través de la implementación de organizadores gráficos en la educación media rural. Revista Assensus, 2(2), 83-98. https://doi.org/10.21897/assensus.1323

Rivas, S. (2018). Acaba Colombia. Motivos para apagar e irnos. Planeta.

Robles, P. (2019). La formación del pensamiento crítico: habilidades básicas, características y modelos de aplicación en contextos innovadores. Rehuso: Revista de Ciencias Humanísticas y Sociales, 4(2), 13-24. https://dialnet.unirioja.es/servlet/articulo?codigo $=7047154$.

Ross, E. W. y Gautreaux, M. (2018). Pensando de manera crítica el pensamiento crítico. Aula Abierta, 47(4), 383-386. https://doi.org/10.17811/rifie.47.4.2018.383-386

Santiago, J. A. (2016). La acción didáctica de las Ciencias Sociales y el desarrollo del pensamiento crítico. Revista Educación y Humanismo, 18(31), 241-256. https://doi.org/10.17081/eduhum.18.31.1377

Sare, A. M. (2019). Pensamiento crítico y su relación con el desempeño docente de las instituciones educativas del nivel secundario del distrito de Cachicadán, provincia Santiago De Chuco año 2019 [tesis de maestría, Universidad César Vallejo]. Universidad César Vallejo Repositorio Digital Institucional. http://repositorio.ucv.edu.pe/handle/20.500.12692/37237

Saussure, F. (1994). Introducción a la lingüística: modelos y reflexiones actuales (2a ed.). Benemérita Universidad Autónoma de Puebla.

Semana. (2020). Los colombianos en tiempos de coronavirus. https://www.semana.com/enfoque/articulo/ los-colombianos-en-tiempos-de-coronavirus/662677.

Schunk, D. H. (2012). Teorías del aprendizaje (6ª ed.). Pearson Educación.

Sorokin, P. A. (1964). Achaques y manías de la sociología moderna y ciencias afines ( $2^{\mathrm{a}}$ ed.). Aguilar.

Suárez, J. R., Pabón, D., Villaveces, L. y Martin, J. A. (2018). Pensamiento crítico y filosofía. Un diálogo con nuevas tonadas. Editorial Universidad del Norte.

Tobón, G. (2018). Estado, política y economía en Colombia —Capitalismo burocrático y gansteril-. Señal Editora.

Torrejano, R. H. (2015). La educación que merecemos no es la educación que tenemos: el problema de la falta de calidad en la educación básica y secundaria en Colombia 1903-1933. Jangwa Pana, 14, 59-74. https://doi.org/10.21676/16574923.1567

Van Dijk, T. (2006). El estudio del discurso. En T. Van Dijk (Comp.), El discurso como estructura y proceso (pp. 21-66). Editorial Gedisa.

Van Gelder, T. (2000). The efficacy of undergraduate critical thinking courses: a survey in progress. Melbourne University Press.

Vega, R. (2015). La universidad de la ignorancia. Capitalismo académico y mercantilización de la educación superior. Ocean Sur.

Villamizar, R. (2016). Nuevas verdades sobre cómo nos gobiernan: compilación actualizada de mis libros. Litocolor. 